\title{
Manipulator Operating With THE TREe- FINGER GRIP FOR FRAGILE CYLINDERS
}

\author{
Golubev, Yu. \& MelKumova, E.
}

Abstract: We consider the problem of fragile cylinders grasping by the manipulator with tree fingers. Each finger contacts the cylinder in a single supporting point with Amontons-Coulomb friction. There is an analogy of the equilibrium of a three-legged robot on a cylinder for the problems of transfer by a manipulator with a three-finger grasp of a fragile cylinders or for robot which legs suspension points are on a cylinder surface. Three supporting points can be due to friction on the opposite sides of the robot center of mass or giving in the dynamics, point $C$. An analogy of the problem is oscillations in the vicinity of the stable equilibrium one cylinder on another. The cylinder lies on one finger rectangular to it, of the hand of a humanoid robot, adheres to the end of the other by two fingers. Similarly holds a glass.

Key words: Tree-finger grasp, Coulomb friction.
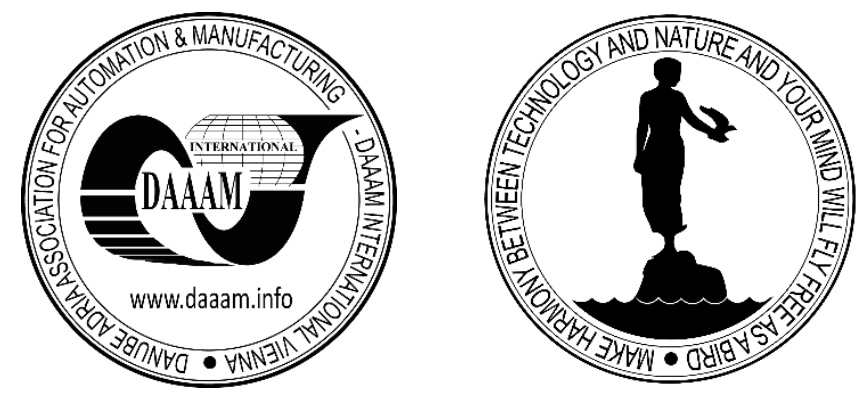

Authors' data: Univ.Prof. Dr.f.-m.n. Golubev, Yu[ry]*; Dr.f.-m.n. Melkumova, E[lena]**, * Keldysh Institute of Applied Mathematics, RAS, Miusskaya sq., 4, 125047, Moscow, Russia, ** Lomonosov Moscow State University,GSP-1, Leninskie Gory, Moscow, 119991, Russia, golubev@keldysh.ru, elena_v_m@mail.ru

This Publication has to be referred as: Golubev, Y[ury] \& Melkumova, E[lena] V[adimovna] (2019). Manipulator Operating with the Tree-finger Grip for Fragile Cylinders, Chapter 35 in DAAAM International Scientific Book 2019, pp.393-400, B. Katalinic (Ed.), Published by DAAAM International, ISBN 978-3-902734-24-2, ISSN 1726-9687, Vienna, Austria

DOI: $10.2507 /$ daaam.scibook.2019.35 


\section{Introduction}

If during driving the car you see the turtle on the road, you save it. But how one robot with a manipulator can help to another. Let the corpus is cylinder. How to hold it. The analytical and the numerical parameter diagrams for two fields of supporting points are shown in this work.

The problem of the distribution of tree fingers reactions was studied. This task arose as a development of [1]. N.E.Zhukovsky investigated equilibrium solid in the plane in the presence of friction [13].

Movement of a six-legged walking apparatus in quasistatic mode planes are investigated in the work of D.E. Okhotsimsky and Yu.F. Golubev [1], using the following criterion static stability of the apparatus. Walking apparatus, leaning o horizontal plane, statically stable in some moment of time if and only if the vertical projection of its center of mass on the supporting surface lies strictly inside the support polygon corresponding to this moment. A natural question arises, what do they look like similar criteria for other surfaces. Including criterion of non-rollover of the robot.

In the work [3] Yu.F. Golubev and I.G. Kolpakova using linear methods programmers have developed an iterative algorithm for distribution of reactions at randomly located points support walking machine. Later, F.L. Chernousko in work [4] cited a series of statements about the conditions of guaranteed equilibrium of the body on rough plane. Study of the organization of the movement of the octopus walking apparatus special design designed for movement in pipes in the presence of friction was carried out in the works [5], [6].

In [7] investigated the conditions of static stability walking robot on a perfect inner surface horizontal cylinder. In [8] the problem was considered for a rough horizontal cylinder, provided that there are no components of the reactions along the the cylinder.

The proposed work continues the study begun by [9], where the problem was considered for a rough horizontal cylinder taking into account reactions along its axis.

The results of solving the equilibrium problem solid under the action of three forces applied to equilibrium biped walking robot on the outer surface of a rough inclined non-holding cylinder. The first contains the statement of the problem. In the second the conditions of equilibrium of a robot under the action of two forces in application to the equilibrium problem of a robot based only on one of the legs. The third is devoted to the balance of the robot, leaning on two legs. The fourth discusses two geometrical equilibrium conditions for a robot based on two legs on top hat.

In this paper, we consider the problem of curved object grasping by the fingers of the robot-manipulator. For example we discussed a three legged humanoid robot with pair of five arms fingers or a monkey-robot with twenty arms and legs fingers. The robot can hold the object by one and grasp by two fingers. An object grasping problem is equivalent to the problem of the walking robot with tree legs. Consider a grasp with $\mathrm{m}$ fingers. Each finger contacts an object in one foothold.

There is an analogy of this problem to the problem of walking robot dynamics on one-side constraint [10]-[14]. 


\section{Main result}

The kinetic moment of the robot relative to the point $O$ is considered. In order for the given motion to be realized, the reactions $\widetilde{\mathbf{R}}_{i}$ at 3 support points must satisfy the following kinetostatic equations [1]:

$$
\sum_{i} \widetilde{\mathbf{R}}_{i}=-\widetilde{\boldsymbol{\Phi}}, \quad \sum_{i} \widetilde{\mathbf{r}}_{i} \times \widetilde{\mathbf{R}}_{i}=-\widetilde{\mathbf{M}}, \quad i=1, \ldots, 3,
$$

where $\widetilde{\mathbf{R}}_{i}$ are the reaction components, $\widetilde{\mathbf{r}}_{i}$ are the radius vectors of the pivot points, $\widetilde{\boldsymbol{\Phi}}$ is the sum of the d'Alembert forces of inertia and external active forces, $\widetilde{\mathbf{M}}$ is the sum of the moments of d'Alembert inertia forces and the moments of external active forces relative to the fixed point $O$.

Assuming that $\widetilde{\boldsymbol{\Phi}}$ is orthogonal $\widetilde{\mathbf{M}}$, we obtain: [8] that the system $\{\widetilde{\boldsymbol{\Phi}}, \widetilde{\mathbf{M}}\}$ can be also used at the point $C$

$$
\tilde{\mathbf{r}}_{C} \times \widetilde{\boldsymbol{\Phi}}=\widetilde{\mathbf{M}}, \quad \mathbf{r}_{C}=-\frac{\widetilde{\mathbf{M}} \times \widetilde{\boldsymbol{\Phi}}}{\widetilde{\boldsymbol{\Phi}}^{2}}, \quad \widetilde{\Phi}=|\widetilde{\boldsymbol{\Phi}}|
$$

where $\mathbf{r}_{C}$ is the vector $\mathbf{O C}$, and $C$ corresponds to point at which the resultant of the reactions is acting. Further problem of reactions distribution $\widetilde{\mathbf{R}}_{i}$ in some fixed point of time is investigated by the proposal that force $\widetilde{\boldsymbol{\Phi}}$ is acting at the point $C$ and force moment there is zero. Motion equations (1) for finding reactions of walking robot body arms and legs prescribed motion can be transformed [10]:

$$
\sum_{i} \widetilde{\mathbf{R}}_{i}=-\widetilde{\boldsymbol{\Phi}}, \quad \sum_{i} \tilde{\mathbf{r}}_{i} \times \widetilde{\mathbf{R}}_{i}=-\tilde{\mathbf{r}}_{C} \times \widetilde{\boldsymbol{\Phi}}
$$

We restrict ourselves to the case when $\widetilde{\boldsymbol{\Phi}} \neq 0$. We bring the system $\{\widetilde{\boldsymbol{\Phi}}, \widetilde{\mathbf{M}}\}$ to the resultant at a point [2]. The point $C$ will also be the point of application of the resultant reactions. For example, a point $C$ may be the center of mass of a transported object.

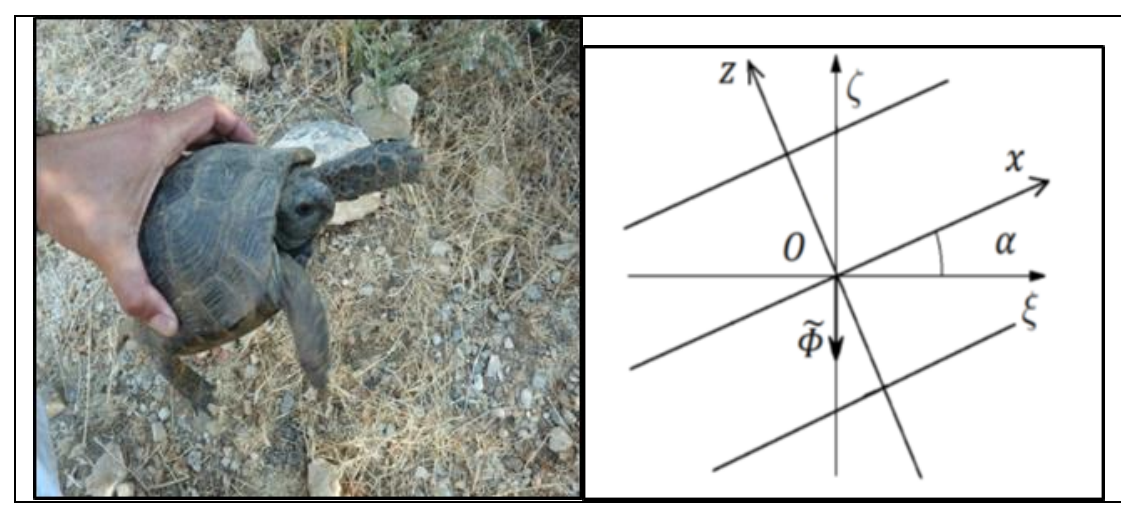

Fig. 1. Holding the cylinder. 
Let the robot support points be located on the surface of a rough straight circular cylinder with a dry friction coefficient $k$, Fig. 1 . And the axis of the cylinder is the angle $\alpha$ with the vector $\widetilde{\boldsymbol{\Phi}}$. In the case of three points of support, the model is supplemented by the introduction of the turning friction moment at the contact points. For three points of support, we restrict ourselves to the consideration of dry friction.

The kinetostatic equations are supplemented by inequalities responsible for getting reactions into the corresponding friction cones. When an object is captured internally, the scalar product of the reaction to the main normal to the surface at the fulcrum should be non-negative: $\widetilde{N}_{i}=\widetilde{\mathbf{R}}_{i} \cdot \mathbf{e}_{i}^{v} \geq 0$, where $\mathbf{e}_{i}^{v}$ is the external normal to the cylinder surface at the $i$-th fulcrum. With external capture, these inequalities are reversed.

Let write the vector $\boldsymbol{r}_{i}$ in the cylinder coordinate: $\boldsymbol{r}_{i}=\left(x_{i},-\sin \varphi_{i}, \cos \varphi_{i}\right)$, $\mathbf{e}_{i}^{v}=\left(0,-\sin \varphi_{i}, \cos \varphi_{i}\right)$, and the vector $\boldsymbol{N}_{i}=\widetilde{\mathbf{N}}_{i} / \widetilde{\Phi}=\left(0,-N_{i} \sin \varphi_{i}, N_{i} \cos \varphi_{i}\right)$, where $\varphi_{i}$ is the angles between axis $O z$ and cylinder normal $\mathbf{e}_{i}^{v}$. We define $\mathbf{e}_{x}$ as the unitary vector in the $O x$ axis, while the vector $\mathbf{e}_{i}^{\tau}=\left(0, \cos \varphi_{i}, \sin \varphi_{i}\right)$ as the tangential to the cylinder. Then the tangential reaction component: $\boldsymbol{F}_{i}=\widetilde{\mathbf{F}}_{i} / \widetilde{\Phi}=$ $\left(F_{i}^{x}, F_{i}^{y z} \cos \varphi_{i}, F_{i}^{y z} \sin \varphi_{i}\right)$, where $F_{i}^{x}=\boldsymbol{F}_{i} \cdot \mathbf{e}_{x}, F_{i}^{y z}=\boldsymbol{F}_{i} \cdot \mathbf{e}_{i}^{\tau}$, the reaction vector $\boldsymbol{R}_{i}=\widetilde{\mathbf{R}}_{i} / \widetilde{\Phi}=\left(R_{i}^{x}, R_{i}^{y}, R_{i}^{z}\right)$, center mass vector $\boldsymbol{r}_{C}=\widetilde{\mathbf{r}}_{C} / \rho=\left(x_{C}, y_{C}, z_{C}\right)$.

We further define the supporting points the coordinate differences along the cylinder, and angles difference: $\Delta x=x_{2}-x_{1}, \Delta \varphi=\varphi_{2}-\varphi_{1}$. We then project system (2) onto the axes $O x y z$. For arbitrary surface we find that the second equation of (2) (corresponding to the angular moment) has the skew-symmetric matrix with respect to the component $R_{i}^{x}$ [11]. These are two independent equations.

If the number of contact points $n+m$ is even, and they are pairwise located at coincident points on opposite sides of a surface of almost zero thickness, then the indicated inequalities are satisfied.

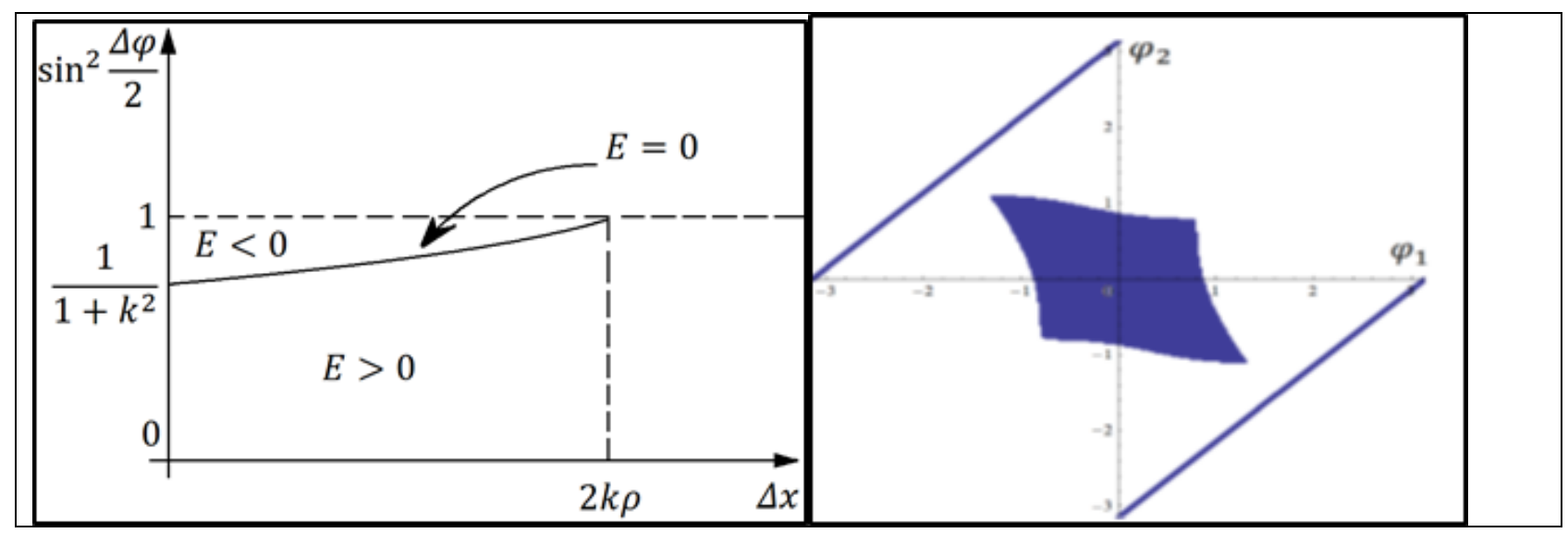

Fig. 2. Regions of parameters for which the problem of the distribution of reactions has a solution.

The second group of inequalities is the condition that the tangent components of the reactions are limited by the limiting value of the Coulomb dry friction. Those. kinetostatic [3] equations are supplemented by quadratic inequalities responsible for getting reactions into the corresponding friction cones. 
As soon as the modules of the tangent reactions exceed the limit value, slipping of the foot or finger relative to the surface occurs. These are quadratic inequalities with respect to the parameter $p$, where $p$ is the difference of the reaction components along the axis of the cylinder, $E$ which coincides and does not depend on $\alpha$ the coefficient $p$ of squared. Note that for an arbitrary surface, the structure and properties of these inequalities are preserved, Fig. 2.

When the robot moves, a change occurs in the one-support and two-support [4] phases. Let $\mathrm{n}=1$. The desired condition for the existence of motion is that the reaction at a single point of support must be equal to the force $\widetilde{\boldsymbol{\Phi}}$ modulo, and the reference point $C$ is located on a straight line along the force $\widetilde{\boldsymbol{\Phi}}$ passing through the point. Moreover, the slope of the line of action of the force $\widetilde{\boldsymbol{\Phi}}$ to the normal should not exceed the angle of friction.

Let $\mathrm{n}=2$. For the bi-support phase, the robot configurations are considered symmetrical with respect to point $\mathrm{C}$ along and across the axis of the cylinder. At $x_{2}=$ $-x_{1}, \varphi_{2}=-\varphi_{1}$, for angles $\alpha=0$ and, $\alpha=\pi / 4$, below, fig. 3. charts are given for areas $E<0$ and $E \geq 0$. These graphs supplement and summarize the results obtained [5], [6].

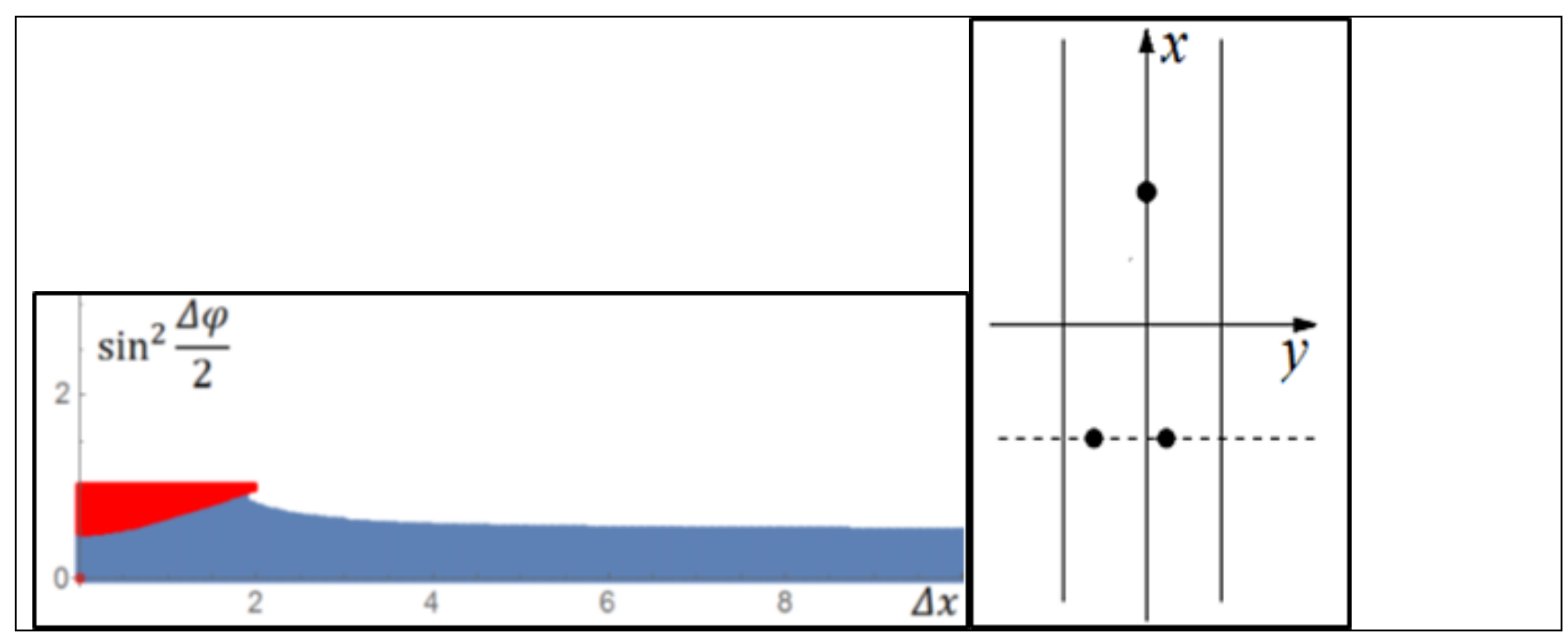

Fig. 3. Symmetrical along the axis of the cylinder configuration of the robot.

For robot finger configurations symmetrical about a point $\mathrm{C}$ along the axis of the cylinder, three cases with a non-negative coefficient $E$ are considered for the distance $x$ between the point $C$ and the reference points: 0,9, 1 and 1,1 for $\rho$ and $k$ equal $1, \alpha$ from 0 to $\pi$ (all 14 different values of the angle of the cylinder) [7], [8]. Areas of existence of a solution to the problem of the distribution of reactions on the plane of two angles corresponding to the projections of the support points on the base of the cylinder and three-dimensional regions complementary to the indicated plane of the applicated point $\mathrm{C}$ are constructed.

When: $\alpha: 0, x: 1$ the area consists of three separately located connected subdomains. On the plane of the angles, each of a pair of parallel lines corresponds to a support in the diametrical plane of the cylinder containing the point $\mathrm{C}$. 
Between these lines is a connected area. It contains a straight line segment corresponding to equal angles, the robot rests from above, on a straight line parallel to the axis of the cylinder, satisfying the restriction on deviations from the direction of the force. The indicated segment on the graphs disappears when $x$ equal to 0,9 for $\alpha$ equal to $\pi / 4$, and when increasing $x$, later, when $4 \pi / 9$ [9], [10]. This corresponds to the fact that the cylinder begins to slide in the grip of the robot with two fulcrum. Valid areas for $\alpha=\pi / 3 ; x=1,1$ built in works [12], [13], [14].

Robot can hold the horizontal cylinder by three fingers. Let one of the points is in vertical plane containing cylinder axis and another are in the plane orthogonal to the axis. Without friction, the cylinder center of mass has to be in the vertical plane that contain the cylinder axis. The supporting points are on the external surface of the lower semi-cylinder and the center mass of the cylinder is in the footholds triangle [13]. If one of supporting points is in the lower semi-cylinder and another are on the upper, the point $C$ has to be out of the footholds triangle.

\section{Conclusion}

During the robot motion, one, two and three supporting point phases are changed. And for example the humanoid robot with five arm fingers can hold the object by one and grasp by two or three-fingers. The reaction distribution problem have a solution in following cases. Let we give some examples.

1. One-supporting point phase. So the motion existing condition is reaction is equal to force and supporting point and the point are on the line along the force. And the angle between the force and the normal not exceed friction angle.

1.1 If the grasp inside the surface then point $C$ is under the surface. In opposite case the grasp is under the surface. Then point $C$ is inside the surface.

1.2 If the number of supporting points is even. And one of each par of the supporting points is on and another is in the thin surface such that we consider them like one geometrical point. Then it does not matter where the point $C$ is on the line.

2. Two-supporting point phases. In case when the grasp is inside the cylinder. The point $C$ and the reactions have to be in the plane parallel to the force.

2.1 If supporting points are on one diameter.

2.2 When coefficient $E<0$. And in some fields with connected set of points, when $E \geq 0$. Robot can hold the cylinder by two fingers on one diameter.

3. Robot can hold the horizontal cylinder by three fingers. Let one of the points is in vertical plane containing cylinder axis and another are in the plane orthogonal to the axis. Without friction, the cylinder center of mass has to be in the vertical plane that contain the cylinder axis. The supporting points are on the external surface of the lower semi-cylinder and the center mass of the cylinder is in the footholds triangle. If the first supporting point is in the lower semi-cylinder and two another are on the upper, the center of mass has to be out of the footholds triangle. So the robot can transfer the cylinder by one, two or three fingers. 


\section{References}

Okhotsimsky, D. \& Golubev, Yu. (1984). Mechanics and motion control of an automatic walking apparatus. M .: Nauka, $312 \mathrm{p}$.

Golubev, Yu. (2019). Fundamentals of Theoretical Mechanics. M .: Moscow State University, $728 \mathrm{p}$.

Golubev, Yu. \& Melkumova, E. (1999.)Static stability conditions of a walking apparatus in a horizontal cylinder and on two planes // Bulletin of the Russian Academy of Sciences. Theory and control systems. No. 2. PP. 116-122.

Melkumova, E. (2000). Oscillations of a walking apparatus around the axis of a smooth supporting cylinder // Moscow University Physics Bulletin. Series 1: Mathematics. Mechanics. No. 5. PP. 47-49.

Golubev, Yu. \& Melkumova, E. (2010). Equilibrium of a biped robot on a rough horizontal cylinder, taking into account reactions along its axis. M.: MAKS Press, 64 p.

Golubev, Yu. \& Melkumova, E. (2012). Walking Robot Dynamics on a Rough Inclined Cylinder. // ESMC - 8th European Solid Mechanics Conference. Graz, Austria, July 913, Book of Abstracts. Ed. Gerhard A. Holzapfel and Ray W. Ogden.

Golubev, Yu. \& Melkumova, E. (2016). Prescribed Motion of a Two-legged Walking Robot on a Rough Cylinder. Proceedings of the International Conference "Stability and Oscillations of Nonlinear Control Systems" (Pyatnitskiy's Conference), Moscow, V.A. Trapeznikov Institute of Control Sciences, PP. 1-4.

Golubev, Yu. \& Melkumova, E. (2016). The existence of a given robot movement when using the pipe as a support. In the collection of the VII All-Russian meetingseminar of the heads of departments and teachers of theoretical mechanics, robotics, mechatronics, universities of the Russian Federation. Materials of the meeting, Makhachkala: Publishing Center "Master", PP. 30-33.

Golubev, Yu. \& Melkumova, E. (2018). Two-legged Walking Robot Prescribed Motion On a Rough Cylinder // AIP Conference Proceedings, v. 1959, No. 030009, PP. 030009-1-030009-8.

Golubev, Yu. \& Melkumova, E. (2018). An Analogy of the Equilibrium of a Twolegged Robot on a Cylinder for the Problem of Transfer by a Manipulator With a Twofinger Grasp of a Cylinder, APM, Proceedings of XLVI Summer School-Conf. "Advanced Problems in Mechanics", June 25-30, St. Petersburg, Russia; Polytech IPME RAS, PP. 117-124, ISSN 2312-9921.

Golubev, Yu. \& Melkumova, E. (2018). Footholds Admissible Areas Structure of a Two-legged Walking Robot on an Inclined Cylinder // Conference Series: Materials Science and Engineering, v. 468, No. 012003, PP. 1-9.

Golubev, Yu. \& Melkumova, E. (2019). Brittle Cylinder Transfer by a Three-finger Grasp. In the collection of the VIII International Conference on Computational Methods for Coupled Problems in Science and Engineering (COUPLED), Sitges (Barcelona), Spain, June 3-5, Technical Program series, place of publication 
Golubev, Y. \& Melkumova, E. V.: Manipulator Operating with the Tree-finger Gri... https://congress.cimne.com /coupled2019/admin/files/fileabstract/a374.pdf, abstract, p. $1-1$

Golubev, Yu. \& Melkumova, E. (2019). Transfer of the Fragile Cylinder with a Twofinger Manipulator in the collection of the XII All-russian Congress on Fundamental Problems of Theoretical and Applied Mechanics. Annotations of reports, place of publication of the RIC of BashSU Ufa, theses, pP. 62-62.

Golubev, Yu., Koryanov, V. \& Melkumova, E. (2019). Bringing an Insectomorphic Robot to Working Condition from an Emergency "Upside Down" Position. in the journal Izvestia of the Russian Academy of Sciences. Theory and Control Systems, No. 6, p. 163-176 DOI: 10.1134/S0002338819060052.

\section{Acknowledgements}

Many thanks to colleagues N.N.Bolotnik, A.M.I.Krivtsov, E.I.Kugushev and V.E.Prynichnikov for advices. 